\title{
CONTRIBUTION OF CRYOMAGNETISM TO SEPARATION OF NON-FERROUS METALS FROM WASTE PRODUCTS
}

\author{
G. GILLET ${ }^{\mathrm{a}, *}$ F. DIOT ${ }^{\mathrm{a}}$ and H. VÉDRINE ${ }^{\mathrm{b}}$ \\ ${ }^{a} I N P L-E N S G$, Laboratoire Environnement et Minéralurgie, Rue du Doyen \\ Roubalt BP 50 - 54501 Vandoeuvre les Nancy, France; \\ ${ }^{\mathrm{b}}$ Bureau de Recherches Géologiques et Minières, SMN/DPEA \\ BP 6009 - 45060 Orleans Cedex 2, France
}

(Received 18 April 1998; Accepted 3 June 1998)

The authors have studied the use of the cryomagnetic separation techniques for the processing of non-ferrous waste since 1989. This paper presents:

- the superconducting magnetic separator and the adapted processing installation;

- the main results achieved by tests with synthetic and industrial effluents containing $\mathrm{Ni}, \mathrm{Cr}, \mathrm{Zn}, \mathrm{Cu}$ or $\mathrm{V}$.

The research work confirmed the assumption that a cryomagnetic separation can be applied to non-ferrous waste materials. Technical difficulties of implementing this technique are understood. A patent containing the innovative process seems to be of common interest for the processing of fluids charged with small amounts of dissolved metals.

As a result of the investigations such an installation appears to be a new way to extract metals from fluids before they are discharged into nature. This is important in view of more stringent controls in the future.

Keywords: HGMS; Cryomagnetism; Heavy metals; Industrial effluent wastes; Hydrated magnetic flocs

\section{INTRODUCTION}

The principle of strong-field/high-gradient magnetic separation is based on production of strong heterogeneities in the originally homogeneous

\footnotetext{
* Corresponding author.
} 
external magnetic field, using small ferromagnetic elements. This results in the force centres of a small range, on which any paramagnetic substances can be trapped [1,2].

The method is based on the use of magnetic attraction forces, which retain paramagnetic particles in a solid/water suspension on magnetized matrix elements (Figs. 1 and 2). Particles with sufficiently high magnetic susceptibility, and non-magnetic particles, i.e. the cleaned product, will flow out of the system. Figure 1 shows how the solid/ water suspension flows through the matrix, whose elements, when exposed to a magnetic field, develop high field gradients immediately around the wires. When the retention capacity of the matrix has been

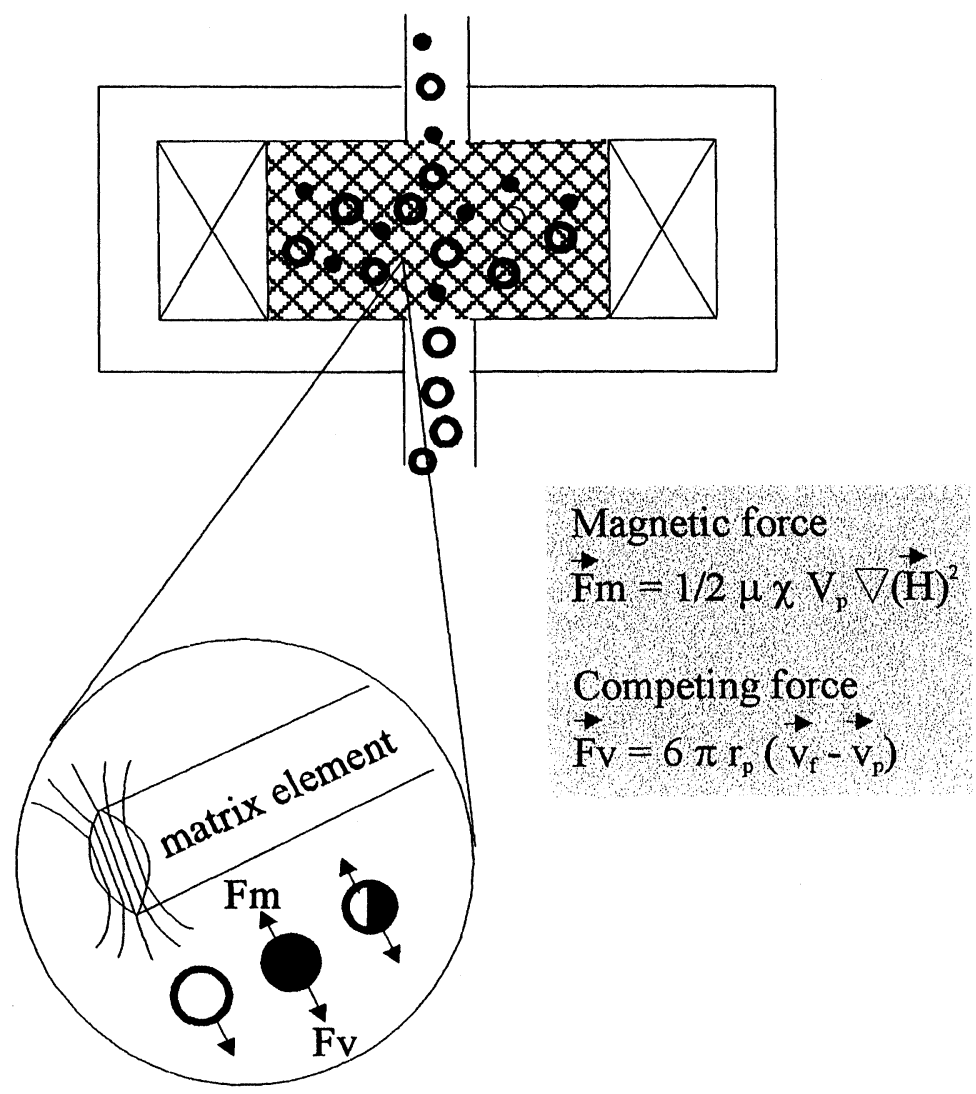

FIGURE 1 Cross section of a filter and attraction onto a magnetized wire. 


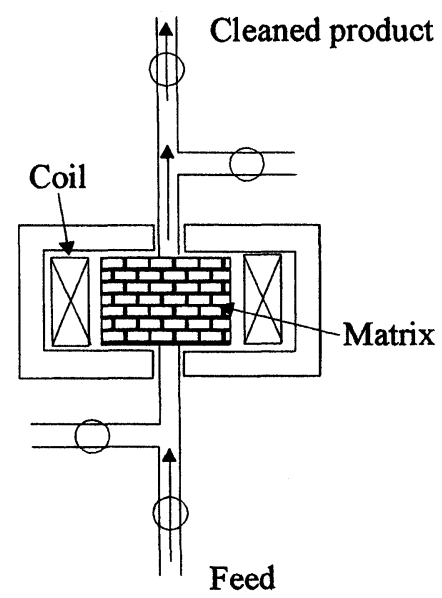

A- Feed in a magnetic field

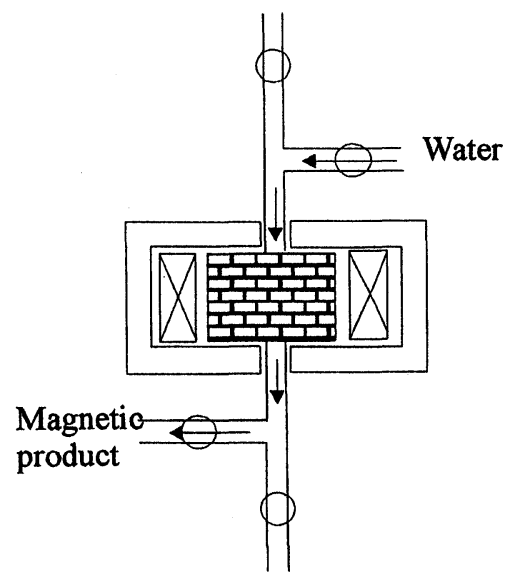

B - Recovery of magnetic product (no magnetic field)

FIGURE 2 The operating principle of high gradient magnetic filter.

reached, suspension feed-flow is stopped and the matrix, still under the magnetic field, is flushed with clean water to eliminate mixed or mechanically trapped particles. After this, the field is switched off and the magnetic particles are removed with a high-pressure water jet (Fig. 2).

\section{CONTRIBUTION AND USE OF SEPARATION WITH SUPERCONDUCTING MAGNETS}

For certain operations of separating ultrafine particles of low magnetic susceptibility, it is necessary to generate magnetic forces of the order of $6 \times 10^{11} \mathrm{~N} \mathrm{~m}^{-3}$ and sometimes even higher. Design of ordinary equipment, materials used for their construction and those of the matrices, make it impossible to reach such high field values, which require that the applied magnetic field is more than $2 \mathrm{~T}$. High electricity consumption, size of the magnetic circuits and the complexity of cooling such installations exclude the possibility of using the usual resistive copper coils. However, new developments and experience acquired in using 
superconducting magnets and their associated cooling systems have opened a new horizon for magnetic separation.

\subsection{The Areas of Application of the Technique}

The technique of magnetic separation with superconducting magnets enables the extraction from a solid/water suspension of superfine, even colloidal, particles that are only weakly magnetic. This technique finds its application in the mineral industry, for purification of coal, industrial minerals, in particular kaolin and talc, etc. It is also of interest in other fields, such as chemistry and biology, and especially the environment.

The application of this technique has enabled the extension of magnetic separation to ores that cannot be economically upgraded by any other means, as well as to completely different fields of activity. It has, in particular, expanded the frontiers of standard separation methods combined with lower operating costs $[3,4]$.

The present areas of application cover:

- the cleaning of products for the glass or ceramics industry that require a high degree of purity, e.g. kaolin or glass sand;

- the cleaning of industrial and urban waste water, and of the cooling water of thermal or nuclear power plants;

- the extraction of contained particles for chemical-synthesis processes, and from energy-transmission fluids or vapours used in electrical power plants;

- the concentration of ultrafine minerals such as $\mathrm{Fe}, \mathrm{Mo}, \mathrm{W}$ or rare earths, or of metallic residues for recycling;

- the processing of used catalysts;

- the field of biochemistry, biology and the food-processing industry.

\subsection{The Testwork Carried Out}

First, a complete processing installation had to be built containing equipment superconducting magnets. Our research then investigated the use of this technique for separation of metals contained in ultrafine products and liquid effluents.

A particular effort was made to clean up dissolved metals from industrial effluents. At present, such metals are removed by precipitation as hydroxides. After decantation and mechanical filtering, the 
sludge is dried in basins or lagoons. Magnetic filtration leads to considerable acceleration of the time needed for separating sludge from liquid, and thus to an increase in the processing rate. In view of the limits to standard magnetic filtration techniques, and of the increasingly strict quality standards set for the discharge of effluents, requiring ever lower concentration thresholds, it was decided to study how superconducting magnetic separation could be applied in this field.

\section{THE INSTALLATION DEVELOPED AS PART OF THE RESEARCH WORK}

\subsection{The Superconducting Magnetic Separator and the Process Used}

The testwork for studying superconducting magnetic separation was carried out using an equipment with extraction matrix and operating in a cyclical fashion, built by the GEC-Alsthom Company (Fig. 3).

A superconducting $\mathrm{Nb}-\mathrm{Ti}$ coil is immersed in a cryostat filled with liquid helium at a temperature of $4.2 \mathrm{~K}$. The canister with its extraction
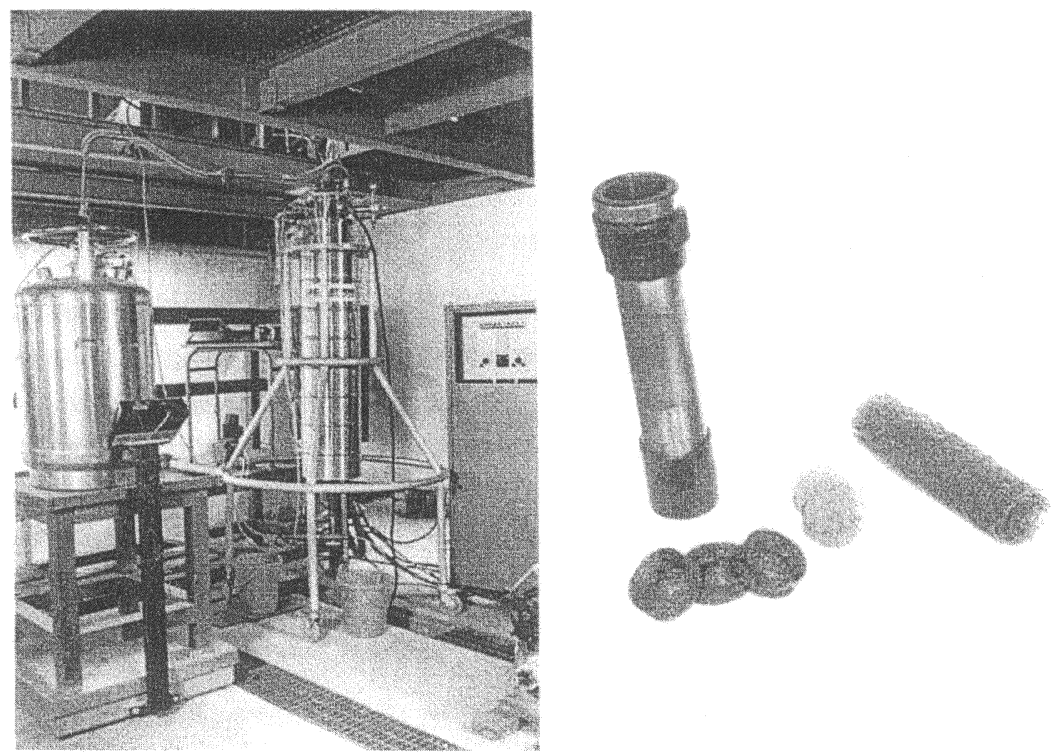

FIGURE 3 L.E.M. Superconducting magnetic separator Canister and matrix types. 
matrix is located in the centre of the cryostat. The matrix consists in fine ferromagnetic elements such as stainless-steel foam or wool, which combines a high porosity with a large surface for trapping. The coil generates a strong magnetic field of $4000 \mathrm{kA} / \mathrm{m}$ with a high field gradient, which converges on the ferromagnetic matrix and thus induces a strong enough magnetic force to trap very small (only a few microns) and/or weakly magnetic particles.

Much R \& D attention was paid to:

- definition of the operating conditions and fine-tuning of the processing system, i.e. the feed modes, operating cycles, recirculation, etc.;

- definition, comparative study of different types of matrix, and selection of the most suitable type.

The final installation as developed is shown in Fig. 4. The feed pump is in the upper part of the system. The pulp to be processed descends through the matrix in a magnetic field. The magnetic particles are trapped by the matrix and the non-magnetic products are recovered, or recycled with a pump for the second purification passage. After this, the magnetic field is switched off and the magnetic products, trapped in the matrix, are removed with a high-pressure water jet for recovery.

\subsection{Purification of Liquid Effluents}

A specific installation was designed as part of this $R$ \& $D$ effort, for the processing of effluents through extraction of precipitated and co-precipitated metals as magnetic "flocs", i.e. magnetic metal hydroxides and co-precipitated magnetic and/or non-magnetic metal hydroxides [1]. Removal of the flocs from the matrix is obtained not by switching off the magnetic field, but by a simple chemical modification caused by rinsing the matrix with acid solution (Fig. 4: 14 and 15). This solution, after having stopped feed-flow, is then charged with metals trapped in the matrix.

The solution, whose volume is limited to that of the canister, can be recirculated for progressive charging with metals and until sufficient metals are contained for hydrometallurgical process. 


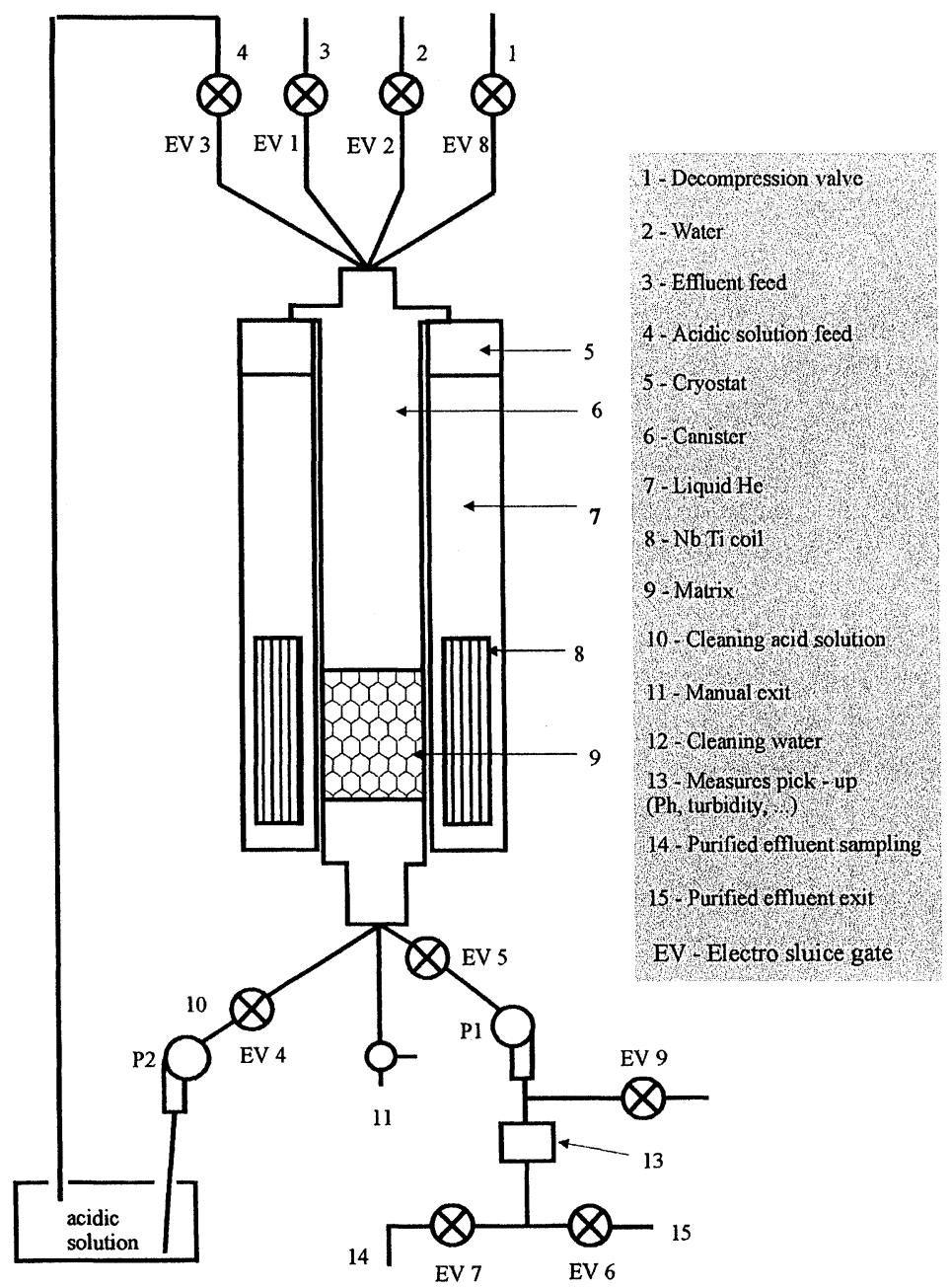

FIGURE 4 Flowsheet of fluid purification with a superconducting magnetic separator.

This process presents several advantages over the "standard" system:

- a smaller volume of waste is produced, which facilitates handling and the later treatment;

- the magnetic field needs no longer to be switched off, which makes coil technology simpler, and reduces the operating cost as less helium is used. 


\section{SEPARATION TESTS AND THE MAIN RESULTS}

\subsection{Search for a Magnetic Carrier for Dissolved Metals}

An interesting way for creating a magnetic support is the co-precipitation of an element to be eliminated with a hydrous iron oxide. The latter should have sufficient magnetic properties for magnetic separation to be efficient.

A major effort was made during the work on this project, to define such hydrous oxides and understand their behaviour. Various modes of metal adsorption on hydrous iron oxides were studied. Figure 5 shows a mixture of ferric chloride and ferrous sulphate, in the proportion of one mole of ferrous iron for two moles of ferric iron (" $\mathrm{Fe}_{3} \mathrm{O}_{4}$ " solution), which led to the precipitation of a hydroxide with strong magnetic properties.

This proportion corresponds to that of magnetite, $\mathrm{Fe}_{3} \mathrm{O}_{4}$, but depending on the amount of iron in solution, the precipitate as identified by XRF analysis could be either magnetite $\left(\mathrm{Fe}_{3} \mathrm{O}_{4}\right)$ or maghemite $\left(\gamma-\mathrm{Fe}_{2} \mathrm{O}_{3}\right)$, the latter precipitating from a solution containing less iron. Maghemite is the unstable phase of hematite $\left(\alpha-\mathrm{Fe}_{2} \mathrm{O}_{3}\right)$, with the spinel structure that, upon drying, shows the magnetite $\left(\mathrm{Fe}_{3} \mathrm{O}_{4}\right)$ spectrum. Depending on the concentration of the iron in solution, the nature of the precipitate will change, but there is also a threshold value beyond which the precipitate will no longer have interesting magnetic
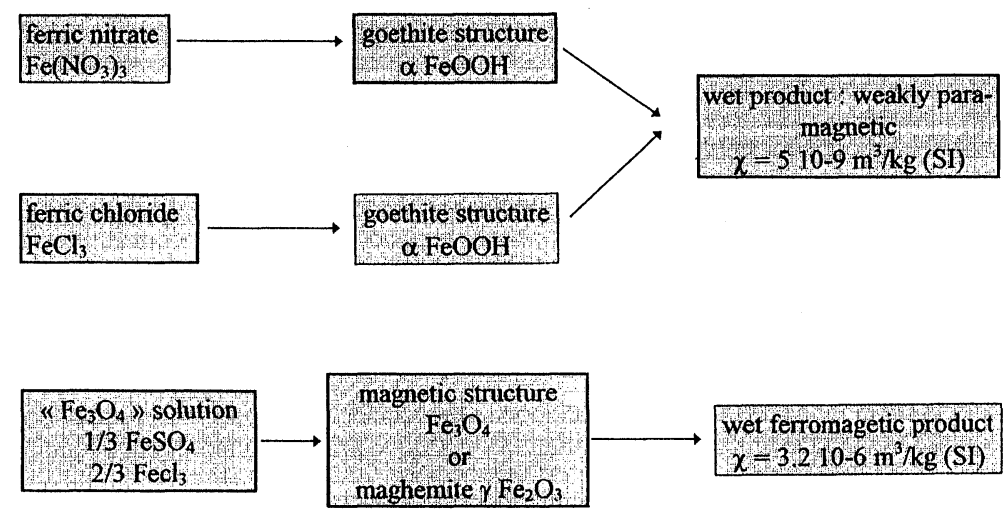

FIGURE 5 Different iron - salt precipitates. 


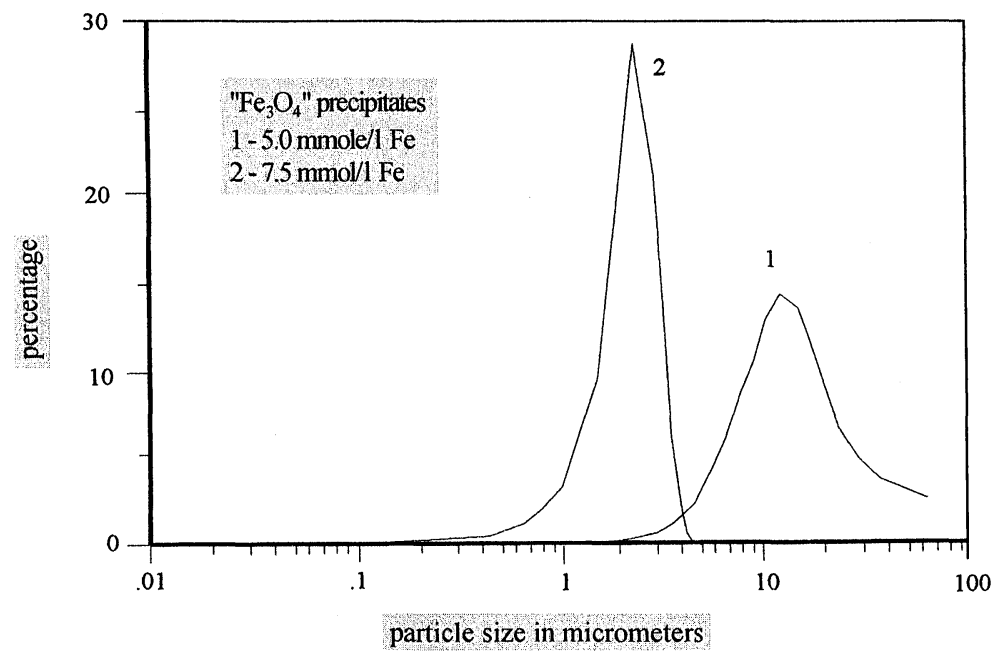

FIGURE 6 Grain size distribution of " $\mathrm{Fe}_{3} \mathrm{O}_{4}$ " solution precipitates.

characteristics. This boundary concentration of iron will vary according to the type and concentration of the metals dissolved in the fluid to be cleaned, which "dilute" the magnetic susceptibility of the end product.

The grain size measurements were made on precipitates only from the " $\mathrm{Fe}_{2} \mathrm{O}_{3}$ " solution (Fig. 6). The curves are seen to be unimodal and fairly symmetric, making it possible to take into account only the $d_{50}$ fraction. The grain size measured is that of the flocs during their passage through the separator after a phase of intense agitation. $\mathrm{A}$ " $\mathrm{Fe}_{3} \mathrm{O}_{4}$ " solution precipitated at $5 \mathrm{mmol} / 1$ of total $\mathrm{Fe}$, shows $d_{50}$ of almost $15 \mu \mathrm{m}$, whereas a more concentrated solution at $7.5 \mathrm{mmol} / \mathrm{l}$ gives $d_{50}$ of $2.4 \mu \mathrm{m}$. Below $7.5 \mathrm{mmol} / 1$ of iron, the precipitate will be a strongly hydrated flocculate with a structure close to that of goethite, as in the case of ferric chloride.

\subsection{Magnetic Cleaning of Precipitates from " $\mathrm{Fe}_{3} \mathrm{O}_{4}$ " Solution}

Filtration tests were carried out with the superconducting magnetic separator. Figure 7 shows, as an example, that with a constant cadmium concentration of $1 \mathrm{mmol} / 1$, the purification percentage increases 
Influence of the relatice iron quantity on magnetic purification of a cadmium solution (constant Cd concentration of $10^{-3} \mathrm{~mol} / 1$

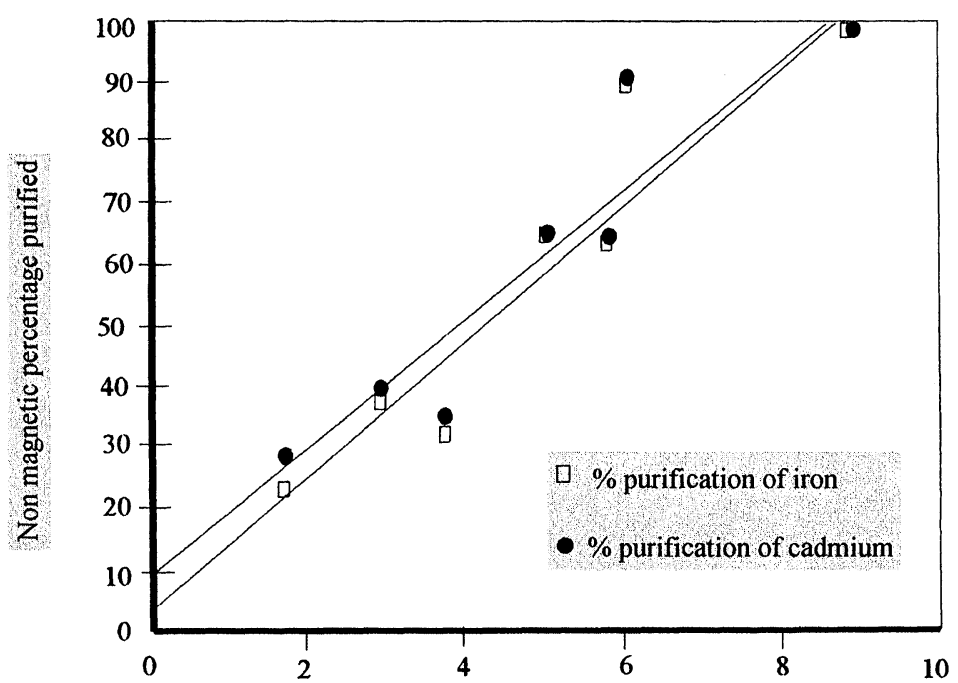

FIGURE 7 Magnetic purification as a function of the iron content of the precipitate.

as a function of the iron content of the precipitate, and that a better than $90 \%$ cleaning rate in one passage is obtained only when the $\mathrm{Fe}_{\mathrm{t}} / \mathrm{Cd}$ molar ratio is over 7.5. A freshy precipitated mixture of $1 \mathrm{mmol} / \mathrm{l}$ cadmium and $7.75 \mathrm{mmol} / \mathrm{l}$ iron has sufficient magnetic susceptibility to be retained in the separator.

Furthermore, it was seen that beyond $2000 \mathrm{kA} / \mathrm{m}(2.5 \mathrm{~T})$, an increase in the magnetic field strength will not measurably improve the efficiency of purification.

For these applications, where magnetic susceptibilities of the flocs are greatly sufficient to be retained at $2.5 \mathrm{~T}$, it is interesting to use, for technical and economic reasons, a cryomagnetic system. Effectively, some economic studies show that cryomagnetic systems supersede advantageously the resistive systems up to $1 \mathrm{~T}$.

All the following tests were carried out at a magnetic field of $5 \mathrm{~T}$ and fluid velocity of $121 / \mathrm{min}$ (the total volume treated was about $50 \mathrm{l}$ by cycle). 


\subsection{Separation Tests on Synthetic Materials}

Several tests were carried out with synthetic materials. Table I shows, as an example, the results obtained with a product containing five metals in solution, i.e. $20 \mathrm{ppm}$ of $\mathrm{Cr}$, and $10 \mathrm{ppm}$ each of $\mathrm{Ni}, \mathrm{Cu}, \mathrm{Fe}$ and $\mathrm{Zn}$. Then the influence of the proportion of " $\mathrm{Fe}_{3} \mathrm{O}_{4}$ " solution added to the solution containing the metals was tested. To be safe, the $\mathrm{pH}$ of the solution was stabilized at 12 by adding $\mathrm{NaOH}$.

By means of simple precipitation of the solution, without adding $\mathrm{Fe}$, the purification for each of the metals is only $40 \%$ for each passage. The recycling of the solution that was pre-purified in the separator does not improve this result; everything that can be magnetically trapped was retained during the first passage. It can be noted, however, that nonmagnetic metals such as zinc or copper are retained in the same manner, thanks to coagulation and adsorption phenomena on the flocs. However, iron and chromium, which have the same purification of $37 \%$, probably precipitate together by substitution of the chromium in ferric hydroxide sites. Above a weighted ratio of 30 for the total iron added to the metals, it can be considered that metal is completely retained in the separator during a single passage.

The purification of zinc appears to be more complex. Because of the amphoteric character of zinc precipitates, both trapping by ferriferous flocs and/or co-precipitation are strongly reduced when the ratio $\mathrm{Fe}$ total/metals is low.

\subsection{Separation Tests on Industrial Effluents}

Treatment of industrial effluents was also studied. Such fluids come mostly from surface-treatment shops, as rinsing water. The main metals in such water can be zinc or copper, depending on its provenance.

TABLE I Magnetic purification of a polymetallic synthetic product

\begin{tabular}{lccccc}
\hline $\begin{array}{l}\text { Mass ratio of } \mathrm{Fe}_{\mathrm{t}} \\
\text { added over the metal } \\
\text { to be eliminated }\end{array}$ & \multicolumn{5}{c}{$\%$ of purified metal after magnetic treatment } \\
\cline { 2 - 6 } & $\mathrm{Fe}$ & $\mathrm{Cr}$ & $\mathrm{Ni}$ & $\mathrm{Zn}$ & $\mathrm{Cu}$ \\
\hline 0 & 37.0 & 37.0 & 45.0 & 32.0 & 42.0 \\
11 & 79.3 & 58.5 & 88.1 & 31.5 & 75.9 \\
31 & 97.4 & 96.6 & $>99$ & 86.3 & $>99$ \\
45 & 99.1 & $>99$ & $>99$ & 83.3 & $>99$ \\
\hline
\end{tabular}


TABLE II Purification results for industrial rinsing fluids

\begin{tabular}{|c|c|c|c|c|c|c|}
\hline \multirow[t]{2}{*}{$\begin{array}{l}\text { Main metal " } M " \\
\text { in the fluid }\end{array}$} & \multicolumn{2}{|c|}{$\begin{array}{c}\text { Concentration before } \\
\text { magnetic processing }(\mathrm{mg} / \mathrm{l})\end{array}$} & \multicolumn{2}{|c|}{$\begin{array}{l}\text { Residual concentration } \\
\text { after magnetic } \\
\text { processing }(\mathrm{mg} / \mathrm{l})\end{array}$} & \multicolumn{2}{|c|}{$\begin{array}{l}\text { Percentage of } \\
\text { purification }\end{array}$} \\
\hline & $M$ & Added Fe & $M e$ & Added Fe & $M e$ & Added $F e$ \\
\hline $\mathrm{Cu}$ & 21.2 & & 0.0 & & 99.81 & 98.70 \\
\hline $\mathrm{Zn}$ & 18.57 & 473.2 & 0.05 & 1.81 & 99.73 & 99.62 \\
\hline V & 38.60 & 720.3 & $<$ Det. Lim. & 5.70 & 100 & 99.21 \\
\hline
\end{tabular}

Fluids from the rinsing of the used vanadium-based catalysers were tested as well.

These different rinsing waters have relatively low concentrations $(20-30 \mathrm{mg} / \mathrm{l})$ of the main dissolved metals, but can contain smaller amounts of other dissolved metals. The latter commonly are not quantified in the characteristics provided by industry, as they are considered to be secondary. Moreover, such rinsing water can contain reagents, in particular the foaming agents, that were necessary for the earlier steps in the industrial process. Each fluid must be subjected to preliminary tests, in order to determine the amount of iron that must be added to obtain a generally magnetic product. It is understood that other impurities that can be precipitated in addition to the dissolved metals, will be trapped in the flocs as well. Table II shows the purification performance obtained for each case.

\section{CONCLUSIONS}

This research and development, has led to confirmation of the interest of cryomagnetic separation as applied to non-ferrous waste materials, as well as to understanding of the technical difficulties of implementing this technique.

It has become clear that the range of applications of such an installation also includes the extraction of metals from fluids that have to be processed before being discharged into nature, in view of even more stringent regulations governing such discharge and the absence of really viable alternative methods for removing such very small amounts of metal. In addition, the standard methods for removing metals involve massive precipitation, implying the disposal of 
large amount of sludge, which is rapidly becoming a problem in its own right.

The process defined in this paper [5] allows to treat important volumes of industrial effluents charged with metals in solution without adding solid or coagulant salt. This technique is of a common interest for the processing of fluids charged with small amounts of dissolved metals, difficult to treat with conventional precipitation and thickening. The flocs recovered in the acid solution represent an important reconcentration of metal that can be possibly treated or recycled.

The arrival of new superconductive materials with a high critical temperature (ceramics) will revolutionize the cryoelectricity market and accelerate the development of this technology and its applications.

\section{Acknowledgements}

The authors thank the Commission of the European Communities DGXII for the partial financial support of this research - EEC contract No. MA2RCT-91-001 (DTEE).

\section{References}

[1] Gillet, G. et al. (1994): "Epuration Cryomagnétique d'Effluents Liquides Industriels". Proceedings of the Colloque International de Recherche sur les Sous-Produits de Traitement et d'Epuration des Eaux. Poitiers, France, 29, 30 September.

[2] Bureau, V. (1993) "Adaptation de la séparation cryomagnétique aux technologies de l'environnement: application à l'épuration d'effluents liquides industriels". INPL Doctorate Thesis, $300 \mathrm{p}$.

[3] Gillet, G. (1991): "Séparation magnétique". Les Techniques de l'Ingénieur - A, Vols. 5220-5222, $32 \mathrm{p}$.

[4] Gillet, G., Bureau, V. and Houot, R. (1992): “Application de la séparation magnétique à l'épuration des eaux urbaines et industrielles". Mines et Carriéres - Les Techniques, pp. 51-61.

[5] Procédé et dispositif d'extraction des métaux paramagnétiques, French Patent (BRGM - INPL) No. 9308000-30-06-93.

[6] Gillet, G., Vedrine, H. et al. (1994): "Cryomagnetic separation of non-ferrous waste". Final Report EEC.

[7] Okud, T., Sugano, I. and Tsuii, T. (1975): "Removal of heavy metals from wastewater by ferrite co-precipitation, Filtration and Separation", 12, 472.

[8] Svoboda, J. (1987): "Magnetic Methods of the Treatment of Minerals Development". Elsevier, Amsterdam 1987.

[9] Watson, J.H.P. and Gilwood, D.C. (1994): "Biomagnetic separation and extraction process for heavy metals metals from solution". Mineral Engineering, 7(8), pp. 10171028 . 


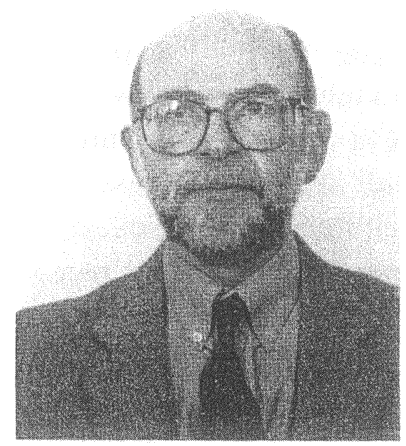

Gérard Gillet was born in 1947 in France, and in 1973 he graduated from Ecole Nationale Supérieure de Géologie (E.N.S.G.) in Nancy. After obtaining his engineering diploma, he was an assistant professor at the School of Geology and worked as the researcher at the Mineral Processing Laboratory of E.N.S.G. $\mathrm{He}$ received his $\mathrm{Ph} . \mathrm{D}$. and his state-thesis in Sciences Physiques in 1975 and 1988. Presently, he is the professor and works on projects in magnetic separation and its applications. Dr. Gérard Gillet is a member of S.M.E.

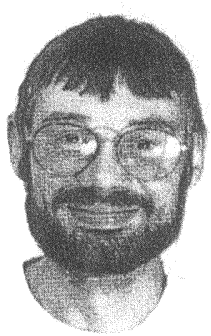

Frédéric Diot was born in 1966. He graduated from I.N.P.L in mineral processing after chemical studies at Reims (France) in 1994. After working as a research engineer in cryomagnetic separation as applied to waste cleaning, he works presently on his $\mathrm{Ph}$.D. subject of which is fine particles separation by magnetic and/or electrostatic methods.

Henri Vedrine is the graduate engineer from Ecole Nationale Supérieure de l'Energie et des Matériaux (E.N.S.E.M.) of the University of Orleans (France). He received his Ph.D. in 1992 from the University of Orleans. He has worked in mineral processing, more specifically in the simulation model of flotation. Presently, he is the researcher in the B.R.G.M. and works in mineral processing and material recycling projects. 